\title{
手性单磷酰保护二胺催化二乙基锌还原三氟甲基酮
}

\author{
王 雪* \\ (扬州工业职业技术学院化学工程学院 江苏扬州 225127)
}

\begin{abstract}
摘要 手性三氟甲基类化合物拥有重要生理活性，为了获得该类化合物，以磷酰胺为配体，乙基锌、三氟甲基芳香醛及 其衍生物为底物合成出一系列的三氟甲基类化合物，所使用的原料廉价易得，催化效率较高. 在最优条件下，可以高 收率、高 $e e$ 值地合成相应的手性三氟甲基类化合物. 尽管配体使用量较高, 但其可回收利用. 最后, 对可能的反应机理 进行了合理推测, 认为反应的高立体选择性主要归因于催化过程中形成的两个六元环过渡态及空间位阻作用.
\end{abstract}

关键词＼cjkstart磷酰胺配体; 不对称催化; 手性三氟甲基类化合物

\section{Reduction of Trifluoromethyl Ketones with Diethyl Zinc Catalyzed by Chiral Monophosphoryl Protected Diamine}

\author{
Wang, Xue* \\ (College of Chemical Engineering, Yangzhou Polytechnic Institute, Yangzhou, Jiangsu 225127)
}

\begin{abstract}
In order to obtain trifluoromethylated organic compounds with important biological activity, using phosphoramide as catalyst, diethylzinc and trifluoromethyl aromatic aldehyde as reactants, chiral trifluoromethylated organic compounds were synthesized through catalytic asymmetric $\beta$-H transfer reduction reaction. The raw materials are cheap and easy to obtain, and the catalytic efficiency is high. The yield and ee value can be guaranteed to be highly and simultaneously under the optimized reaction conditions. Despite the large amount of catalyst, the ligand is very convenient to recycle and reuse in this system. At the same time, the reaction mechanism was speculated, and it was considered that the high stereoselectivity of the reaction was due to the formation of two six membered ring transition states and steric hindrance.

Keywords phosphoramide ligand; asymmetric catalysis; chiral trifluoromethylated compound
\end{abstract}

由于含有三氟甲基的化合物的具有特殊属性及反 应活性，该类化合物在现代医学及农药产品中起着不可 替代的作用 ${ }^{[1]}$, 例如治疗抑有症的贝氟沙通(1) ${ }^{[2]}$ 、特效 除草剂氟乐灵(2) $)^{[3]}$ 、治疗艾滋病的依法韦仑 $(3)^{[4]}$ 、治疗 佝偻病的氟骨三醇 $(4)^{[5]}$ 以及治疗类癌瘤综合征的 Telotristat Etiprate (5)(图 1) ${ }^{[6]}$ 等. 由于人们生活中对光学 纯的三氟甲基类化合物的需求量越来越大, 这极大地促 进了人们对其不对称合成的研究. 在已开发的方法中, 最为常见的合成路径是对三氟甲基酮类化合物进行不 对称催化还原, 常见的有过渡金属催化加氢 [7]、生物催 化还原 ${ }^{[8]}$ 、手性有机金属试剂还原 ${ }^{[9]}$ 、不对称硼烷还原 ${ }^{[10]}$ 以及不对称 $\beta$ 氢还原反应 ${ }^{[11]}$.

目前, 使用不对称 $\beta$ 氢还原方法的报道 ${ }^{[1]]}$ 比较少. 2002 年, Chong 等 ${ }^{[11]}$ 报道了使用不对称 $\beta$ 氢还原三氟甲
基酮化合物来合成高手性的三氟甲基类化合物，合成过 程中使用了手性金属美试剂作为催化剂，该方法可以以 均大于 $95 \%$ 的收率和 $e e$ 值得到产物, 但是由于镁试剂 的高反应活性，该反应必须将反应温度控制在较低($78{ }^{\circ} \mathrm{C}$ ). 乙基锌试剂是较好的 $\beta$ 氢供体, 已被 Higashiyama 等 ${ }^{[12]}$ 证实可用来还原三氟甲基酮，其他团队也发 现二乙基锌可以还原三氟甲基酮 ${ }^{[13]}$ ，但是使用二乙基 锌的 $\beta$ 氢不对称还原 $\alpha$-三甲基酮的报道比较少 ${ }^{[14]}$.

众所周知，在多个可还原基团存在的条件下，选择 性地还原其中的一个或部分基团非常困难. 选择性地还 原甲基/三氟甲基二酮化合物的方法报道较少 ${ }^{[15]}$, 主要 原因是 $\alpha$-甲基嶡基和 $\alpha$-三氟甲基羰基的化学性质非常 相似, 因此在还原过程中非常容易同时被还原. 因此,

\footnotetext{
* Corresponding author. E-mail: lovexue-angel@163.com

Received February 26, 2021; revised April 2, 2021; published online June 7, 2021.

Project supported by the Outstanding Teaching Team of 2019 "Blue Project" in Jiangsu Colleges and Universities (No. 2019-69).

江苏高校 2019 年 “蓝色工程” 优秀教学团队(No. 2019-69)资助项目.
} 
<smiles>COC[C@H]1CN(c2ccc(OCC[C@H](O)C(F)(F)F)cc2)C(=O)O1</smiles>

1

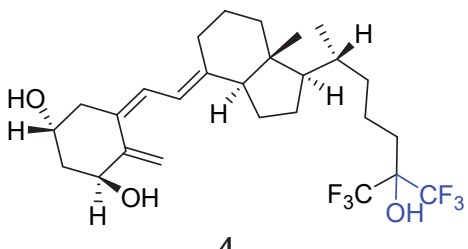<smiles>CCCN(CCC)c1c([N+](=O)[O-])cc(C(F)(F)F)cc1[N+](=O)[O-]</smiles>

2<smiles>C[C@]1(C#CC2CC2)OC(=O)Nc2ccc(Cl)cc21</smiles>

3<smiles>CCOC(=O)C(N)Cc1ccc(-c2cc(OC(c3ccc(Cl)cc3-n3ccc(C)n3)C(F)(F)F)nc(N)n2)cc1</smiles>

5

图 1 含有三氟甲基构架的药物举例

Figure 1 Examples of drugs containing trifluoromethyl framework

寻找能够高效高选择性地还原甲基和三氟甲基二酮的 方法迫在眉睫.

Huang 等[16]曾报道过许多以手性磷酰胺类配体作 为催化剂催化锌试剂的不对称催化反应. 本工作采用以 $(R, R)-1,2$-环己二胺为原料合成的手性磷酰胺配体 9a (Scheme 1)作为催化剂不对称催化二乙基锌与 $\alpha$-三氟甲 基酮发生 $\beta$ 氢还原反应，当甲基酮和三氟甲基酮同时存 在时，该反应可选择性地催化还原三氟甲基酮.<smiles>NC1CCCCC1N</smiles><smiles>CCCNCCOc1ccccc1</smiles>
$0^{\circ} \mathrm{C} \sim$ r.t., $4 \mathrm{~h}$<smiles>O=C1c2ccccc2C(=O)N1C1CCCCC1NP(=O)(O)P</smiles>
$\underset{\text { reflux, } 2 \mathrm{~h}}{\stackrel{\mathrm{N}_{2} \mathrm{H}_{4} \cdot \mathrm{H}_{2} \mathrm{O}, \mathrm{EtOH}}{\longrightarrow}}$<smiles>N[C@@H]1CCCC[C@H]1NP(=O)=P</smiles>

8

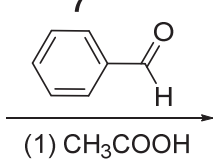

(2) $\mathrm{NaBH}_{3} \mathrm{CN}$

图式 1 配体 9a 合成路线

Scheme 1 Synthesis route of ligand 9a

\section{1 结果与讨论}

\section{1 磷酰胺配体的篮选}

为了篮选得到最佳配体，以 $\alpha$-三氟甲基苯甲酮和二 乙基锌为底物, 对化合物 9 11 进行篎选. 在 $0{ }^{\circ} \mathrm{C}$, 配 体的用量为 $20 \mathrm{~mol} \%$, 反应时间为 $5 \mathrm{~h}$ 的条件下进行催 化 $\beta$ 氢还原反应, 结果列于表 1. 表中数据显示, 当用 $\mathbf{1 0}$ 作为配体时，所得化合物 12a 的收率虽然可达到 77\%, 但其 $e e$ 值仅有 $48 \%$ (Entry 1); 当使用 11 作为配体时, 所 得化合物 $12 \mathbf{a}$ 的收率均在 $20 \%$ 左右, 且 $e e$ 值也仅有 $10 \%$
表 1 手性配体的篮选 ${ }^{a}$

Table 1 Screening of chiral ligand

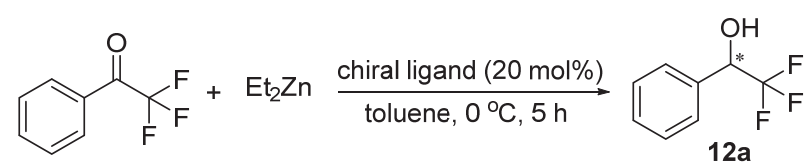
$12 a$

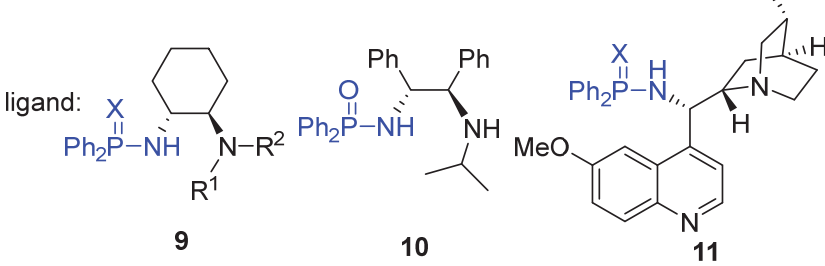

\begin{tabular}{cllccccc}
\hline Entry & Ligand & $\mathrm{X}$ & $\mathrm{R}^{1}$ & $\mathrm{R}^{2}$ & Yield/\% & $e e^{b} / \%$ & Config. $^{c}$ \\
\hline 1 & $\mathbf{1 0}^{[16 \mathrm{~d}]}$ & - & - & - & 77 & 48 & $R$ \\
2 & $\mathbf{1 1 a}^{[16 \mathrm{c}]}$ & $\mathrm{O}$ & - & - & 20 & 12 & $S$ \\
3 & $\mathbf{1 1 b}^{[16 \mathrm{c}]}$ & $\mathrm{S}$ & - & - & 19 & 11 & $S$ \\
$\mathbf{4}$ & $\mathbf{9 a}$ & $\mathbf{O}$ & $\mathbf{B n}$ & $\mathbf{B n}$ & $\mathbf{9 6}$ & $\mathbf{5 5}$ & $\boldsymbol{R}$ \\
5 & $\mathbf{9 b}^{[16 \mathrm{a}]}$ & $\mathrm{S}$ & $\mathrm{Bn}$ & $\mathrm{Bn}$ & 93 & 48 & $R$ \\
6 & $\mathbf{9 c}^{[16 \mathrm{a}]}$ & $\mathrm{O}$ & $\mathrm{Et}$ & $\mathrm{H}$ & 75 & 8 & $R$ \\
7 & $\mathbf{9 d}^{[16 \mathrm{a}]}$ & $\mathrm{O}$ & $i$-Pr & $\mathrm{H}$ & 88 & 19 & $S$ \\
\hline
\end{tabular}

${ }^{a}$ All reactions were carried out with benzaldehyde on a $0.5 \mathrm{mmol}$ scale; ${ }^{b}$ Isolated yields; ${ }^{c}$ Determined by chiral $\mathrm{GC}$ analysis and the absolute configuration was assigned by comparison of the sign of specific rotation value with the literature value.

左右(Entries 2, 3); 当使用 9a 为配体时, 得到化合物 12a 的收率可达到 96\%, 且 $e e$ 值可达到 55\% (Entry 4); 当以 9b 作为催化剂时, 所得 12a 的收率与 9a 差别不大, 但 $e e$ 值差别较大 (表 1, Entry 5); 当用 $\mathrm{N}$ 上取代基更换为烷 基取代基得到的配体 9c 和 9d 催化该反应时，收率尚可, 但 $e e$ 值很低(表 1, Entries 6,7). 由此可发现在配体 9a 存 在下, 所得产物在兼顾收率的同时还可得到较高 $e e$ 值.

\section{2 最佳反应条件的篮选}

之后对最佳反应条件进行了摸索，分别对乙基锌的 用量、配体的用量、反应溶剂、反应时间以及反应温度 进行了篮选(表 2). 当锌试剂的使用量从 0.8 equiv. 增高 
为 3.0 equiv. 时, 所得 12a 的收率呈上升趋势, 但 $e e$ 值呈 先升高后降低趋势 (表 2, Entries 1 4), 综合考虑收率与 $e e$ 值因素, 将锌试剂的使用量定为 2.0 equiv. (表 2, Entries 1 4). 在此基础上把配体 9a 的用量由 $20 \mathrm{~mol} \%$ 提 高到 $30 \mathrm{~mol} \%$, 结果表明 $e e$ 值有少许提高, 收率却有较 大的下降, 考虑到配体使用的经济性, 优选配体使用量 为 $20 \mathrm{~mol} \%$ (表 2, Entries 3, 5). 接着又对反应使用的溶 剂进行了笛选, 分别考察了正己烷、二氯甲烷及甲苯(表 2 , Entries 3, 6, 7), 结果表明以甲苯为溶剂时收率及 $e e$ 值 均为最大值, 优选甲苯作为溶剂. 之后又对反应的时间 进行了篮选, 结果表明随着时间的不断延长, 收率无明 显变化但 $e e$ 值却有明显的增高, 最后优选反应时间为 $24 \mathrm{~h}$ (表 2, Entries 3, 8, 9). 最后对反应温度进行了篮选, 结果表明温度越低, 反应的收率缓慢降低, 但 $e e$ 值却有 明显增高趋势(表 2, Entries 9 11), 因此反应温度最终 选择一 $20{ }^{\circ} \mathrm{C}$. 由此得出最佳反应条件为: 乙基锌的使 用量为 2.0 equiv., 配体使用量为 $20 \mathrm{~mol} \%$, 溶剂为甲苯, 反应时间为 $24 \mathrm{~h}$, 反应温度为 $-20{ }^{\circ} \mathrm{C}$.

表 2 反应条件的优化 ${ }^{a}$

Table 2 Optimization of the reaction conditions

\begin{tabular}{|c|c|c|c|c|c|c|c|}
\hline Entry & $Y$ & $Z$ & Solvent & Temp. $/{ }^{\circ} \mathrm{C}$ & Time $/ \mathrm{h}$ & Yeild $^{b} / \%$ & $e e^{c / \%}$ \\
\hline 1 & 0.8 & 20 & Hexane & 0 & 5 & 31 & 22 \\
\hline 2 & 1.5 & 20 & Hexane & 0 & 5 & 90 & 53 \\
\hline 3 & 2.0 & 20 & Hexane & 0 & 5 & 93 & 51 \\
\hline 4 & 3.0 & 20 & Hexane & 0 & 5 & 97 & 28 \\
\hline 5 & 2.0 & 30 & Hexane & 0 & 5 & 89 & 54 \\
\hline 6 & 2.0 & 20 & $\mathrm{CH}_{2} \mathrm{Cl}_{2}$ & 0 & 5 & 84 & 55 \\
\hline 7 & 2.0 & 20 & Toluene & 0 & 5 & 95 & 54 \\
\hline 8 & 2.0 & 20 & Toluene & 0 & 12 & 96 & 63 \\
\hline 9 & 2.0 & 20 & Toluene & 0 & 24 & 94 & 77 \\
\hline 10 & 2.0 & 20 & Toluene & -10 & 24 & 92 & 80 \\
\hline 11 & 2.0 & 20 & Toluene & -20 & 24 & 91 & 88 \\
\hline
\end{tabular}

${ }^{a}$ All reactions were carried out with benzaldehyde on a $0.5 \mathrm{mmol}$ scale; ${ }^{b}$ Isolated yields; ${ }^{c}$ Determined by chiral $\mathrm{GC}$ analysis and the absolute configuration was assigned by comparison of the sign of specific rotation value with the literature value.

\section{3 底物的拓展}

确定了最优反应条件后, 为了验证以上催化条件普 适性, 对反应的底物进行了扩展, 实验结果如表 3. 当 底物为对位被 $\mathrm{F} 、 \mathrm{Cl} 、 \mathrm{Br} 、 \mathrm{Me}$ 取代的 $\alpha$-三氟甲基苯甲 酮时, 所得 $\alpha$-三氟甲基化合物的收率均在 70\%以上，且 $e e$ 值在 $60 \%$ 以上(表 3, Entries 2 5). 当把酮中的苯环更 换为萗环时, 得到的 $\alpha$-三氟甲基-2-䒺醇收率为 $90 \%$, 但 $e e$ 值稍低, 仅有 $53 \%$ (表 3, Entry 6); 当改变卤素的种类, 将 $\alpha$-三氟甲基苯甲酮中三氟甲基中的一个氟原子更换
表 3 底物的拓展 $a$

Table 3 Expansion of the substrate

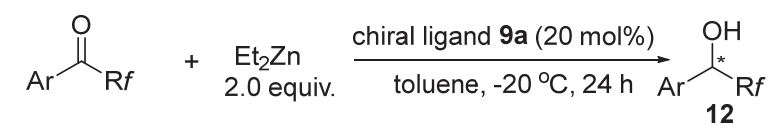

12 (Config.) $\quad \mathrm{Yield}^{a} / \%$ e

$\overline{{ }^{a}}$ All reactions were carried out with benzaldehyde on a $0.5 \mathrm{mmol}$ scale; ${ }^{b}$ Isolated yields; ${ }^{c}$ Determined by chiral $\mathrm{GC}$ analysis and the absolute configuration was assigned by comparison of the sign of specific rotation value with the literature value.

为氯原子后, 所得产物的收率及 $e e$ 值均比较高(表 3 , Entry 7); 当以 $\alpha$-三氟甲基对乙酰基苯甲酮为原料进行 实验时, $\beta$ 氢还原可选择性地在 $\alpha$-三氟甲基酮处进行, 乙酰基处几乎不发生还原反应，且所得醇的 $e e$ 值也可 高达 70\%以上(表 3, Entry 8); 最后使用邻间位分别被 $\mathrm{Br}$ 或 $\mathrm{F}$ 原子取代的芳香醛为底物合成相应的 $\mathbf{1 2}$ 时, 收率均 
在 $80 \%$ 左右，除了间氟或间溴取代化合物 12 的 $e e$ 值在 $80 \%$ 以下，其他的均在 $80 \%$ 以上(表 3, Entries 9～12).

\section{4 机理推测}

最后, 为了说明配体 9a 的不对称催化效果, 我们基 于 Ishihara 的路易斯酸碱理论对反应机理进行了相应的 推测, 认为在发生还原反应时分子间形成的两个六元环 及空间位阻作用导致反应选择性地生成手性专一的 $\alpha$ 三氟甲基化合物(Scheme 2). 当乙基锌与酮发生 $\beta$ 氢还 原时, 羰基上的 $\mathrm{C}$ 和 $\mathrm{O}$ 与二乙基锌中一个乙基上的 $\alpha$ 碳、 $\beta$ 氢以及锌形成第一个六元环状过渡态; 底物醛中 的氧原子、乙基锌试剂中的锌原子、磷酰胺配体中的磷 原子、氧原子、氮原子和另一分子的锌形成第二个六元 环. 当醛中的三氟甲基与配体 9a 中的苯环在同一侧时, 三氟甲基中的氟原子可与苯环上的氢原子形成氢键, 更 加稳定, 但当醛中的苯环与配体 9a 中的苯环在一侧时, 无法形成分子间氢键且存在较大的空间位阻, 不稳定, 因此更倾向于生成单一构型的醇.
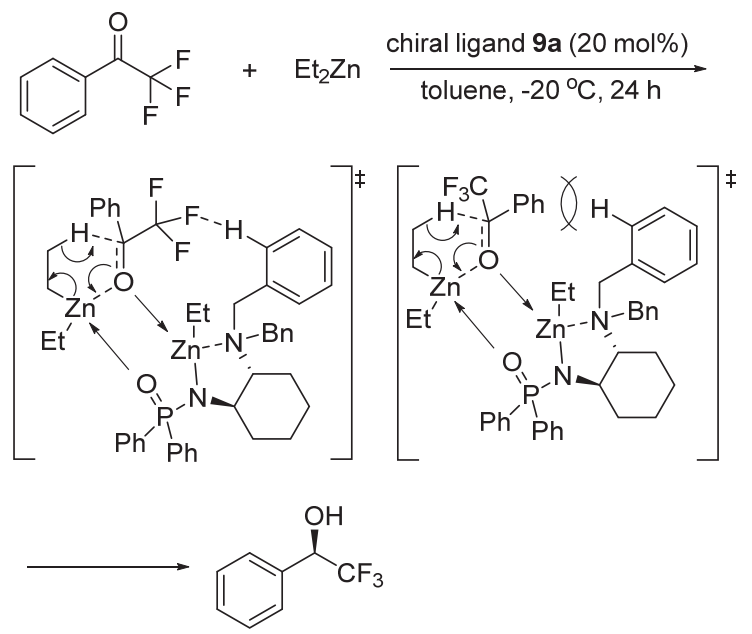

图式 2 反应机理推测

Scheme 2 Speculation of reaction mechanism

\section{2 结论}

以廉价易得的 $(R, R)-1,2$-环己二胺为原料经过几步 简单反应获得了手性磷酰胺配体 9a, 以该配体作为催 化剂催化不对称 $\beta$ 氢还原反应, 可以高效、高选择性地 合成具有重要医用价值的 $\alpha$-三氟甲基化合物. 配体 9a 的普适性较广, 合成步骤少, 合成方法简单, 使用的原 料廉价易得, 虽然在催化过程中配体的使用量比较大, 但该配体能够回收再利用, 且回收后的配体与新制备的 配体拥有相同的催化效果. 最后对反应机理进行了较为 合理的解释. 配体在催化反应过程中形成的两个六元环 状过渡态以及空间位阻作用是所得三氟甲基类化合物 拥有较高的 $e e$ 值的原因，另外当甲基酮和三氟甲基酮
同时存在时，该体系可选择性地催化还原三氟甲基酮.

\section{3 实验部分}

\section{1 仪器与试剂}

$(R, R)-1,2$-环己二胺购于上海泰坦科技股份有限公 司; 一水合对甲苯磺酸、邻苯二甲酸䣶及水合肼均从上 海阿拉丁生化科技股份有限公司购买; 二乙基锌试剂从 萨恩化学技术(上海)有限公司进行购买; 其他试剂均购 于上海泰坦科技股份有限公司.

气相色谱仪(浙江福立仪器公司, 9790II 型); 核磁共 振谱仪(Bruker 公司, Bruker AVANCE III $400 \mathrm{MHz}$ 型), 以 $\mathrm{CDCl}_{3}$ 为溶剂; 超高分辨质谱仪 (Bruker 公司, Impact II UHR-TOF 型); 高效液相色谱仪 (日本岛津, LC20ATvp 型). 旋光仪 (上海精科有限公司, SGW-1 型); GC 数据以 FID 检测器和手性 GC 柱(Chirasil Dex CB column, $25 \mathrm{~m} \times 0.32 \mathrm{~mm} \times 0.25 \mathrm{~mm}$ film thickness)的进行检 测得到; HPLC 数据采用手性柱(如 AD-H 柱、AS-H 柱等) 测试得到.

\section{2 实验方法}

\subsection{1 配体 9a 的合成}

向反应瓶中加入 $50 \mathrm{~mL}$ 甲苯及一水合对甲苯磺酸 $(9.51 \mathrm{~g}, 50 \mathrm{mmol})$, 加热至 $120{ }^{\circ} \mathrm{C}$, 加热回流过程中及 时将水分分出, 搅拌 $2 \mathrm{~h}$ 后停止加热, 缓慢降温至室温 后加入 $(R, R)$-1,2-环已基二胺 $(5.71 \mathrm{~g}, 50 \mathrm{mmol})$ 和邻苯二 甲酸䣶 $(7.41 \mathrm{~g}, 50 \mathrm{mmol})$, 加入过程不断搅拌. 加毕继续 升温至回流并保持回流分水 $6 \mathrm{~h}$. 反应结束后停止加热, 缓慢降温至室温后进行抽滤并收集滤饼. 依次将饱和碳 酸钠溶液 $(100 \mathrm{~mL})$ 和上述滤饼加入到反应瓶中，摚拌至 固体全溶. 所得溶液用二氯甲烷萃取 $(80 \mathrm{~mL} \times 3)$, 收集 有机相, 在搅拌条件下用无水硫酸钠干燥有机相, 过滤, 滤液经减压浓缩, 得到单氨基保护的 $(R, R)-1,2$-环己基二 胺. 得到的产品不再进行其他后处理, 直接用于下一步 反应.

控温在 $0 \sim 5{ }^{\circ} \mathrm{C}$, 用二氯甲烷 $(250 \mathrm{~mL})$ 将上步反应 所得粗品溶解并将溶液加入到反应瓶中, 再加入三乙胺 $(15.18 \mathrm{~g}, 150 \mathrm{mmol})$, 控温 $0 \sim 5{ }^{\circ} \mathrm{C}$ 搅拌 $30 \mathrm{~min}$. 将二苯 基膦酰氯(23.65 g, $100 \mathrm{mmol}$ )溶解于二氯甲烷 $(250 \mathrm{~mL})$ 备用. 控温 $0 \sim 5{ }^{\circ} \mathrm{C}$ 将上述二苯基膦酰氯溶液缓慢加入 反应瓶，缓慢升温至室温并摚拌反应 $4 \mathrm{~h}$. 将反应液冷 却至 $0 \sim 5{ }^{\circ} \mathrm{C}$, 用纯化水 $(80 \mathrm{~mL})$ 淬灭反应, 用二氯甲烷 萃取 $(100 \mathrm{~mL} \times 3)$, 合并有机相, 在搅拌条件下用无水硫 酸钠干燥有机相, 过滤, 滤液经减压浓缩得粗品, 采用 柱层析法对粗品进行分离纯化 [流动相: 乙酸乙酯/正己 烷, $V: V=1: 2)$ ], 最终得 $19.11 \mathrm{~g} N-((1 R, 2 R)-2-(1,3-二$ 氧异吲哚啉-2-基)环已基)- $P, P$-二苯基次膦酰胺(7). 淡 
黄色固体, 产率 $86 \%$. m.p. $193 \sim 195{ }^{\circ} \mathrm{C} ;[\alpha]_{\mathrm{D}}^{20}-41.5(c$ 2.00, $\mathrm{CH}_{2} \mathrm{Cl}_{2}$ ); ${ }^{1} \mathrm{H} \mathrm{NMR}\left(400 \mathrm{MHz}, \mathrm{CDCl}_{3}\right) \delta: 1.21 \sim 1.40$ $(\mathrm{m}, 3 \mathrm{H}), 1.64 \sim 1.86(\mathrm{~m}, 3 \mathrm{H}), 2.24 \sim 2.39(\mathrm{~m}, 2 \mathrm{H}), 2.92 \sim$ $3.03(\mathrm{~m}, 1 \mathrm{H}), 3.48 \sim 3.67(\mathrm{~m}, 1 \mathrm{H}), 3.84 \sim 4.03(\mathrm{~m}, 1 \mathrm{H})$, $7.01 \sim 7.13(\mathrm{~m}, 2 \mathrm{H}), 7.24 \sim 7.32(\mathrm{~m}, 1 \mathrm{H}), 7.30 \sim 7.41(\mathrm{~m}$, $2 \mathrm{H}), 7.42 \sim 7.53(\mathrm{~m}, 3 \mathrm{H}), 7.63 \sim 7.75(\mathrm{~m}, 4 \mathrm{H}), 7.78 \sim 7.85$ $(\mathrm{m}, 2 \mathrm{H}) ;{ }^{13} \mathrm{C}$ NMR $\left(100 \mathrm{MHz}, \mathrm{CDCl}_{3}\right) \delta: 24.4,24.9,28.4$, $37.2(\mathrm{~d}, J=2.5 \mathrm{~Hz}), 51.4,56.5$ (d, $J=5.9 \mathrm{~Hz}), 122.7,128.2$ $(\mathrm{d}, J=12.8 \mathrm{~Hz}), 128.1$ (d, $J=12.6 \mathrm{~Hz}), 131.3(\mathrm{~d}, J=38.4$ $\mathrm{Hz}), 131.2(\mathrm{~d}, \quad=9.5 \mathrm{~Hz}), 131.8(\mathrm{~d}, J=7.6 \mathrm{~Hz}), 131.9(\mathrm{~d}$, $J=9.1 \mathrm{~Hz}), 133.3$ (d, $J=5.8 \mathrm{~Hz}), 133.4,168.6,168.4 ;{ }^{31} \mathrm{P}$ NMR (162 MHz, $\mathrm{CDCl}_{3}$ ) $\delta: 22.3$; HRMS (ESI) calcd for $\mathrm{C}_{26} \mathrm{H}_{26} \mathrm{~N}_{2} \mathrm{O}_{3} \mathrm{P}[\mathrm{M}+\mathrm{H}]^{+}$445.4708, found 445.5721.

依次将无水乙醇(500 mL)及所得化合物 7 (19.11 g, $43 \mathrm{mmol}$ )加入反应瓶中, 室温搅拌至全溶, 室温下将水 合肼 $(43 \mathrm{~mL})$ 缓慢滴加至反应瓶中, 再热至回流反应 $2 \mathrm{~h}$. 反应结束后, 将反应体系缓慢降温至室温, 减压浓缩得 浓缩物, 依次向浓缩物中加入二氯甲烷 $(1000 \mathrm{~mL})$ 和乙 醚(1000 mL), 加毕室温搅拌 $30 \mathrm{~min}$, 过滤得滤液, 在摚 拌条件下用无水硫酸钠干燥有机相, 过滤, 滤液经减压 浓缩得单异丙基取代的 $(R, R)$-1,2-环己基二胺 8 粗品, 不 再进行其他处理, 直接进行下一步反应.

依次向反应瓶中加入无水甲醇 $430 \mathrm{~mL}$ 、所得产物 8 粗品以及 $4 \AA$ 分子笁 $1.9 \mathrm{~g}$, 室温摚拌下再依次向反应瓶 中加入冰醋酸 $(2.0 \mathrm{~mL})$ 和苯甲醛 $(10.04 \mathrm{~g}, 94.6 \mathrm{mmol})$, 继 续室温反应, 用薄层色谱(TLC)检测反应, 直至无苯甲 醛的剩余. 控温 $0 \sim 5{ }^{\circ} \mathrm{C}$, 搅拌条件下加入 $\mathrm{NaBH}_{3} \mathrm{CN}$ (16.25 g, $258 \mathrm{mmol}$ ), 再缓慢升温至室温, 继续反应 10 $\mathrm{h}$, 反应结束后过滤, 滤液经减压浓缩, 所得浓缩物溶解 于二氯甲烷 $(100 \mathrm{~mL})$, 有机相先使用饱和碳酸钠溶液洗 涤 (100 mL), 再使用无水硫酸钠搅拌干燥, 过滤, 滤液 再减压浓缩, 浓缩物使用柱层析 [流动相: $\mathrm{MeOH} /$ $\mathrm{CH}_{2} \mathrm{Cl}_{2}, V: V=1: 40$ ]进行分离纯化, 得 $13.04 \mathrm{~g}$ (产率 $75 \%)$ 淡黄色固体 $N-((1 R, 2 R)-2-($ 二芳基氨基) 环己 基)- $P, P$-二苯基次膦酰胺(9a). m.p. $132 \sim 134{ }^{\circ} \mathrm{C}$; $[\alpha]_{\mathrm{D}}^{20}$ -11.7 (c 1.00, $\mathrm{CH}_{2} \mathrm{Cl}_{2}$ ); ${ }^{1} \mathrm{H}$ NMR (400 MHz, $\left.\mathrm{CDCl}_{3}\right) \delta$ : $0.83 \sim 1.23(\mathrm{~m}, 3 \mathrm{H}), 1.25 \sim 1.38(\mathrm{~m}, 1 \mathrm{H}), 1.42 \sim 1.53(\mathrm{~m}$, $1 \mathrm{H}), 1.72 \sim 1.83(\mathrm{~m}, 1 \mathrm{H}), 1.95 \sim 2.12(\mathrm{~m}, 2 \mathrm{H}), 2.32 \sim 2.50$ $(\mathrm{m}, 1 \mathrm{H}), 3.23 \sim 3.49(\mathrm{~m}, 3 \mathrm{H}), 3.63 \sim 3.91(\mathrm{~m}, 2 \mathrm{H}), 4.58 \sim$ $4.73(\mathrm{~m}, 1 \mathrm{H}), 7.08 \sim 7.26(\mathrm{~m}, 9 \mathrm{H}), 7.36 \sim 7.58(\mathrm{~m}, 7 \mathrm{H})$, $7.70 \sim 7.84(\mathrm{~m}, 4 \mathrm{H}) ;{ }^{13} \mathrm{C} \mathrm{NMR}\left(100 \mathrm{MHz}, \mathrm{CDCl}_{3}\right) \delta: 22.5$, $24.4,25.5,33.5,51.8,53.1,62.5,62.6,127.0,127.9,128.1$, $128.2,128.3,128.4,128.6,129.1,129.7,131.2,131.3$, $131.5,131.6,131.8,132.1,132.2,133.7,134.4,135.0$, 135.7, 139.6; ${ }^{31} \mathrm{P}$ NMR (162 MHz, $\left.\mathrm{CDCl}_{3}\right) \delta: 21.1$; HRMS
(ESI) calcd for $\mathrm{C}_{32} \mathrm{H}_{36} \mathrm{~N}_{2} \mathrm{OP}[\mathrm{M}+\mathrm{H}]^{+}$495.2487, found 496.2475.

\section{2 .2 催化还原三甲基酮的基本操作}

向反应瓶加入溶剂 $(3 \mathrm{~mL}) 、$ 磷酰胺配体 $(0.10 \mathrm{mmol})$ 、 二乙基锌试剂及搅拌子, 用氮气换气 3 次, 在室温下搅 拌反应 $5 \mathrm{~min}$ 后将反应管放于低温反应槽中搅拌，在搅 拌条件下使用注射器将相应的芳香醛 $(0.5 \mathrm{mmol})$ 注入反 应管中, 控温一 $20{ }^{\circ} \mathrm{C}$ 反应 $24 \mathrm{~h}$. 反应结束, 在 $-40{ }^{\circ} \mathrm{C}$ 条件下向反应管中逐滴滴加 $1 \mathrm{~mol} / \mathrm{L}$ 的稀盐酸 $(5 \mathrm{~mL})$ 淬 灭反应，再用乙酸乙酯 $(10 \mathrm{~mL} \times 3)$ 萃取，合并有机相并 用无水硫酸镁干燥, 过滤, 滤液经减压浓缩得到相应的 粗品, 粗品经柱层析(流动相: 乙酸乙酯/正己烷, $V$ : $V=1: 10)$ 分离纯化，得到相应的 $\alpha$-三氟甲基化合物 12 . 通过高效液相色谱法(HPLC)或气相色谱法(GC)对相应 产物的 $e e$ 值进行测试. 分子构型通过旋光仪进行旋光 度的测试并与文献报道相对比得出.

$(R)$-三氟苯乙醇 $(\mathbf{1 2 a})^{[14]}$ : 淡黄色液体，产率 $91 \%$, $88 \%$ ee [手性 HPLC 检测, 柱温: $40{ }^{\circ} \mathrm{C}, t_{\mathrm{R}}=17.7 \mathrm{~min}(R)$, $\left.t_{\mathrm{R}}=18.4 \mathrm{~min}(S)\right] .{ }^{1} \mathrm{H} \mathrm{NMR}\left(400 \mathrm{MHz}, \mathrm{CDCl}_{3}\right) \delta: 2.74(\mathrm{~s}$, $1 \mathrm{H}), 5.01(\mathrm{q}, J=6.6 \mathrm{~Hz}, 1 \mathrm{H}), 7.35 \sim 7.52(\mathrm{~m}, 5 \mathrm{H}) ;{ }^{13} \mathrm{C}$ NMR (100 MHz, $\left.\mathrm{CDCl}_{3}\right) \delta: 72.7$ (q, $\left.J=32.2 \mathrm{~Hz}\right), 124.5$ (q, $J=281.0 \mathrm{~Hz}), 127.4,128.1,129.5,134.1$.

$(R)$-对氟三氟苯乙醇 $(\mathbf{1 2 b})^{[14]}$ : 淡黄色液体, 产率 $77 \%, 60 \% e e$ [手性 HPLLC, 柱温: $40{ }^{\circ} \mathrm{C}, t_{\mathrm{R}}=10.4 \mathrm{~min}$ $\left.(S), t_{\mathrm{R}}=10.7 \mathrm{~min}(R)\right] .{ }^{1} \mathrm{H} \mathrm{NMR}\left(400 \mathrm{MHz}, \mathrm{CDCl}_{3}\right) \delta: 3.14$ $(\mathrm{s}, 1 \mathrm{H}), 5.03(\mathrm{q}, J=6.5 \mathrm{~Hz}, 1 \mathrm{H}), 7.02 \sim 7.15(\mathrm{~m}, 2 \mathrm{H})$, $7.35 \sim 7.54(\mathrm{~m}, 2 \mathrm{H}) ;{ }^{13} \mathrm{C} \mathrm{NMR}\left(100 \mathrm{MHz}, \mathrm{CDCl}_{3}\right) \delta: 72.5$ (q, $J=32.2 \mathrm{~Hz}), 115.7$ (d, $J=21.9 \mathrm{~Hz}), 123.9$ (q, $J=282.1$ Hz), 129.4 (d, $J=8.2 \mathrm{~Hz}), 129.8,163.2$ (d, $J=248.9 \mathrm{~Hz})$.

$(R)$-对氯三氟苯乙醇 $(12 c)^{[14]}$ : 淡黄色色液体, 产率 $73 \%, 63 \% e e\left[\right.$ 手性 HPLC, 柱温: $40{ }^{\circ} \mathrm{C}, t_{\mathrm{R}}=8.0 \mathrm{~min}(S)$, $\left.t_{\mathrm{R}}=8.6 \mathrm{~min}(R)\right] .{ }^{1} \mathrm{H} \mathrm{NMR}\left(400 \mathrm{MHz}, \mathrm{CDCl}_{3}\right) \delta: 3.02(\mathrm{~s}$, $1 \mathrm{H}), 5.03$ (q, $J=6.6 \mathrm{~Hz}, 1 \mathrm{H}), 7.31 \sim 7.44(\mathrm{~m}, 4 \mathrm{H}) ;{ }^{13} \mathrm{C}$ NMR (100 MHz, $\left.\mathrm{CDCl}_{3}\right) \delta: 72.3$ (q, $\left.J=32.0 \mathrm{~Hz}\right), 124.2$ (q, $J=283.5 \mathrm{~Hz}), 128.5,129.6,132.4,135.6$.

$(R)$-对溴三氟苯乙醇 $(\mathbf{1 2 d})^{[18]}$ : 类白色固体, 产率 $71 \%, 66 \% e e$ [手性 HPLC, 柱温: $40{ }^{\circ} \mathrm{C}, t_{\mathrm{R}}=12.7 \mathrm{~min}(R)$, $\left.t_{\mathrm{R}}=13.9 \min (S)\right]$. m.p. $49 \sim 51{ }^{\circ} \mathrm{C} ;{ }^{1} \mathrm{H} \mathrm{NMR}(400 \mathrm{MHz}$, $\left.\mathrm{CDCl}_{3}\right) \delta: 2.82(\mathrm{~d}, J=4.4 \mathrm{~Hz}, 1 \mathrm{H}), 4.96 \sim 5.01(\mathrm{~m}, 1 \mathrm{H})$, $7.32(\mathrm{~d}, J=8.2 \mathrm{~Hz}, 2 \mathrm{H}), 7.54 \sim 7.58(\mathrm{~m}, 2 \mathrm{H}) ;{ }^{13} \mathrm{C} \mathrm{NMR}$ $\left(100 \mathrm{MHz} \mathrm{CDCl}_{3}\right) \delta: 72.4(\mathrm{q}, J=32 \mathrm{~Hz}), 123.7,123.9$ (q, $J=281 \mathrm{~Hz}), 129.1,132.7,132.5$.

$(R)$-对甲基三氟苯乙醇 $(12 \mathrm{e})^{[14]}$ : 淡黄色固体，产率 $76 \%, 67 \% e e$ [手性 HPLC, 柱温: $40{ }^{\circ} \mathrm{C}, t_{\mathrm{R}}=14.6 \min (R)$, $\left.t_{\mathrm{R}}=16.4 \min (S)\right]$. m.p. $43 \sim 45{ }^{\circ} \mathrm{C} ;{ }^{1} \mathrm{H}$ NMR $(400 \mathrm{MHz}$, 
$\left.\mathrm{CDCl}_{3}\right) \delta: 2.43(\mathrm{~s}, 3 \mathrm{H}), 2.88(\mathrm{br}, 1 \mathrm{H}), 4.95 \sim 5.02(\mathrm{~m}, 1 \mathrm{H})$, $7.15 \sim 7.31(\mathrm{~m}, 2 \mathrm{H}), 7.35 \sim 7.45(\mathrm{~m}, 2 \mathrm{H}) ;{ }^{13} \mathrm{C}$ NMR $(100$ $\left.\mathrm{MHz}, \mathrm{CDCl}_{3}\right) \delta: 21.2,72.5(\mathrm{q}, J=31.8 \mathrm{~Hz}), 124.2(\mathrm{q}, J=$ $281.1 \mathrm{~Hz}), 127.5,129.1,131.5,139.5$.

$(R)$-2-菜三氟苯乙醇 $(\mathbf{1 2 f})^{[14]}$ : 类白色固体, 产率 $90 \%, 53 \% e e$ [手性 HPLC, 柱温: $40{ }^{\circ} \mathrm{C}, t_{\mathrm{R}}=10.4 \mathrm{~min}(S)$, $\left.t_{\mathrm{R}}=10.8 \min (R)\right]$. m.p. $81 \sim 83{ }^{\circ} \mathrm{C} ;{ }^{1} \mathrm{H}$ NMR $(400 \mathrm{MHz}$, $\left.\mathrm{CDCl}_{3}\right) \delta: 3.35(\mathrm{~s}, 1 \mathrm{H}), 5.18(\mathrm{q}, J=6.8 \mathrm{~Hz}, 1 \mathrm{H}), 7.43 \sim$ $7.67(\mathrm{~m}, 3 \mathrm{H}), 7.81 \sim 8.02(\mathrm{~m}, 4 \mathrm{H}) ;{ }^{13} \mathrm{C}$ NMR $(100 \mathrm{MHz}$, $\left.\mathrm{CDCl}_{3}\right) \delta: 72.9$ (q, $\left.J=31.8 \mathrm{~Hz}\right), 124.5,124.3(\mathrm{q}, J=280.0$ Hz), 126.5, 126.5, 127.5, 127.6, 128.2, 128.8, 131.6, 133.2, 133.5.

$(R)-2,2-$ 二氟-2-氯-1-苯基乙醇 $(\mathbf{1 2 g})^{[14]}$ : 淡黄色液体, 产率 91\%, 76\% ee[手性 HPLC, 柱温: $40{ }^{\circ} \mathrm{C}, t_{\mathrm{R}}=16.1$ $\left.\min (R), t_{\mathrm{R}}=21.4 \min (S)\right] ;{ }^{1} \mathrm{H}$ NMR $\left(400 \mathrm{MHz}, \mathrm{CDCl}_{3}\right) \delta$ : $3.04(\mathrm{~s}, 1 \mathrm{H}), 5.05(\mathrm{t}, J=6.6 \mathrm{~Hz}, 1 \mathrm{H}), 7.31 \sim 7.60(\mathrm{~m}, 5 \mathrm{H})$; ${ }^{13} \mathrm{C}$ NMR $\left(100 \mathrm{MHz}, \mathrm{CDCl}_{3}\right) \delta: 77.5,127.5,128.6,128.9$ (t, $J=297.0 \mathrm{~Hz}), 129.3,132.0,134.5$.

$(R)$-对乙酰基三氟苯乙醇 $(\mathbf{1 2 h}){ }^{[14]}$ : 淡黄色液体, 产 率 89\%, 73\% ee[手性 HPLC, 柱温: $40{ }^{\circ} \mathrm{C}, t_{\mathrm{R}}=8.3 \mathrm{~min}$ $\left.(S), t_{\mathrm{R}}=9.1 \mathrm{~min}(R)\right] ;{ }^{1} \mathrm{H} \mathrm{NMR}\left(400 \mathrm{MHz}, \mathrm{CDCl}_{3}\right) \delta: 2.60$ $(\mathrm{s}, 3 \mathrm{H}), 3.22(\mathrm{~s}, 1 \mathrm{H}), 5.08 \sim 5.10(\mathrm{~m}, 1 \mathrm{H}), 7.51 \sim 7.63(\mathrm{~m}$, $2 \mathrm{H}), 7.91 \sim 8.03(\mathrm{~m}, 2 \mathrm{H}) ;{ }^{13} \mathrm{C} \mathrm{NMR}\left(100 \mathrm{MHz}, \mathrm{CDCl}_{3}\right) \delta$ : 26.4, 72.3 (q, $J=32.1 \mathrm{~Hz}), 124.0$ (q, $J=282.3 \mathrm{~Hz}), 127.5$, $128.6137 .8,139.3,198.5$.

$(R)$-邻澳三氟苯乙醇 $(\mathbf{1 2 i})^{[19]}$ : 淡黄色液体, 产率 $85 \%, 91 \% e e$ [手性 HPLC, 柱温: $40{ }^{\circ} \mathrm{C}, t_{\mathrm{R}}=10.3 \min (R)$, $\left.t_{\mathrm{R}}=12.7 \mathrm{~min}(S)\right] .{ }^{1} \mathrm{H}$ NMR $\left(400 \mathrm{MHz}, \mathrm{CDCl}_{3}\right) \delta: 7.57(\mathrm{~d}$, $J=8.4 \mathrm{~Hz}, 2 \mathrm{H}), 7.34$ (d, $J=8.3 \mathrm{~Hz}, 2 \mathrm{H}), 4.97$ (q, $J=6.4$ $\mathrm{Hz}, 1 \mathrm{H}), 2.63$ (s, $1 \mathrm{H}) ;{ }^{13} \mathrm{C}$ NMR $\left(100 \mathrm{MHz}, \mathrm{CDCl}_{3}\right) \delta$ : 133.1, 132.1, 129.4, 124.2 (q, $J=282.2 \mathrm{~Hz}), 123.7,72.5$ (q, $J=32.2 \mathrm{~Hz})$.

$(R)$ - 间溴三氟苯乙醇 $(\mathbf{1 2} \mathbf{j})^{[19]}$ : 淡黄色液体, 产率 $81 \%, 78 \% e e$ [手性 HPLC, 柱温: $40{ }^{\circ} \mathrm{C}, t_{\mathrm{R}}=12.0 \mathrm{~min}(R)$, $\left.t_{\mathrm{R}}=14.8 \mathrm{~min}(S)\right] .{ }^{1} \mathrm{H} \mathrm{NMR}\left(400 \mathrm{MHz}, \mathrm{CDCl}_{3}\right) \delta: 7.65(\mathrm{~s}$, $1 \mathrm{H}), 7.55 \sim 7.48(\mathrm{~m}, 1 \mathrm{H}), 7.40(\mathrm{~d}, J=7.6 \mathrm{~Hz}, 1 \mathrm{H}), 7.25(\mathrm{t}$, $J=7.8 \mathrm{~Hz}, 1 \mathrm{H}), 5.02 \sim 4.98(\mathrm{~m}, 1 \mathrm{H}), 3.03(\mathrm{~d}, J=4.4 \mathrm{~Hz}$, $1 \mathrm{H}) ;{ }^{13} \mathrm{C}$ NMR $\left(100 \mathrm{MHz}, \mathrm{CDCl}_{3}\right) \delta$ : 136.3, 130.3, 124.2 (q, $J=281.1 \mathrm{~Hz}), 123.4,116.5,114.9,114.3,72.0$ (q, $J=$ $32.1 \mathrm{~Hz})$.

$(S)$ - 邻氟三氟苯乙醇 $(\mathbf{1 2} \mathbf{k})^{[20]}$ : 淡黄色固体, 产率 $76 \%, 87 \%$ ee [手性 HPLC, 柱温: $40{ }^{\circ} \mathrm{C}, t_{\mathrm{R}}=10.3 \mathrm{~min}(R)$, $\left.t_{\mathrm{R}}=12.7 \mathrm{~min}(S)\right]$. m.p. $49 \sim 51{ }^{\circ} \mathrm{C} ;{ }^{1} \mathrm{H}$ NMR $(400 \mathrm{MHz}$, $\left.\mathrm{CDCl}_{3}\right) \delta: 3.84(\mathrm{~s}, 1 \mathrm{H}), 5.40(\mathrm{q}, J=6.60 \mathrm{~Hz}, 1 \mathrm{H}), 7.04 \sim$ $7.18(\mathrm{~m}, 1 \mathrm{H}), 7.17 \sim 7.21(\mathrm{~m}, 1 \mathrm{H}), 7.33 \sim 7.42(\mathrm{~m}, 1 \mathrm{H})$, $7.63(\mathrm{t}, J=7.34 \mathrm{~Hz}, 1 \mathrm{H}) ;{ }^{13} \mathrm{C} \mathrm{NMR}\left(100 \mathrm{MHz}, \mathrm{CDCl}_{3}\right) \delta$ : $115.5(\mathrm{~d}, J=21.3 \mathrm{~Hz}), 120.2 \sim 128.9$ (m), 121.5 (d, $J=$ $11.7 \mathrm{~Hz}), 124.8$ (d, $J=3.7 \mathrm{~Hz}), 128.6(\mathrm{~d}, J=2.9 \mathrm{~Hz}), 131.4$ (d, $J=8.8 \mathrm{~Hz}), 160.6(\mathrm{~d}, J=248.0 \mathrm{~Hz})$.

$(S)$-间氟三氟苯乙醇 $(121)^{[18]}$ : 无色液体，产率 $85 \%$, $62 \% e e$ [手性 HPLC, 柱温: $40{ }^{\circ} \mathrm{C}, t_{\mathrm{R}}=8.0 \mathrm{~min}(R), t_{\mathrm{R}}=$ $8.6 \mathrm{~min}(S)] .{ }^{1} \mathrm{H}$ NMR $\left(400 \mathrm{MHz}, \mathrm{CDCl}_{3}\right) \delta: 2.94(\mathrm{~s}, 1 \mathrm{H})$, $5.05(\mathrm{q}, J=6.5 \mathrm{~Hz}, 1 \mathrm{H}), 7.05 \sim 7.19(\mathrm{~m}, 1 \mathrm{H}), 7.22 \sim 7.32$ $(\mathrm{m}, 2 \mathrm{H}), 7.33 \sim 7.45(\mathrm{~m}, 1 \mathrm{H}) ;{ }^{13} \mathrm{C}$ NMR $(100 \mathrm{MHz}$, $\left.\mathrm{CDCl}_{3}\right) \delta: 72.1(\mathrm{q}, J=32.5 \mathrm{~Hz}), 114.5(\mathrm{~d}, J=23.0 \mathrm{~Hz})$, $116.4(\mathrm{~d}, J=21.2 \mathrm{~Hz}), 123.2(\mathrm{~d}, J=2.2 \mathrm{~Hz}), 124.2$ (q, $J=$ $282.1 \mathrm{~Hz}), 130.4(\mathrm{~d}, J=8.1 \mathrm{~Hz}), 136.3(\mathrm{~d}, J=7.3 \mathrm{~Hz})$, $162.9(\mathrm{~d}, J=246.9 \mathrm{~Hz})$.

辅助材料(Supporting Information) 化合物 7、9a、10、 11 和 12 的 ${ }^{1} \mathrm{H} \mathrm{NMR}$ 及 ${ }^{13} \mathrm{C} \mathrm{NMR}$ 图谱, 以及所得三氟甲 基化合物 12 的 GC 或 HPLC 图谱数据. 这些材料可以免 费从本刊网站(http://sioc-journal.cn/)上下载.

\section{References}

[1] (a) Xu, W. Y.; Feng, Y. S. Chem. J. Chin. Univ. 2020, 41, 1567 (in Chinese).

(徐文艺, 冯乙巳，高等学校化学学报, 2020, 41, 1567.)

(b) Hu, Y. L.; Yang, T. Y.; Deng, Z. B.; Wang, K. H.; Li, P. F.; Huang, D. F.; Su, Y. P. J. Org. Chem. 2020, 85,12304.

(c) Xie, Q. Q.; Hu, J. B. Chin. J. Chem. 2020, 38, 202.

(d) Schlosser, M. Angew. Chem., Int. Ed. 2006, 45, 5432.

(e) Muller, K.; Faeh, C.; Diederich, F. Science 2007, 317, 1881.

(f) Purser, S.; Moore, P. R.; Swallow, S.; Gouverneur, V. Chem. Soc. Rev. 2008, 37, 320

(g) Kirk, K. L. Org. Process Res. Dev. 2008, 12, 305

[2] Shibatomi, K.; Narayama, A.; Abe Y.; Iwasa, S. Chem. Commun. 2012, 48, 7380 .

[3] Endeshaw, M. M.; Li, C.; Leon, J. D.; Yao, N.; Latibeaudiere, K.; Premalatha, K.; Morrissette, N.; Werbovetz, K. Bioorg. Med. Chem. Lett. 2010, 20, 5179.

[4] Plosker, G. L.; Perry, C. M.; Goa, K. L. Pharmacoeconomics 2001, 19,421

[5] Ikeda, M.; Takahashi, K.; Dan, A.; Koyama, K.; Kubota, K.; Tanaka, T.; Hayashi, M. Bioorg. Med. Chem. 2000, 8, 2157.

[6] Bednarz, M. S.; Paul, S. D.; Kanamarlapudi, R. C.; Perlberg, A.; Zhang, H. M. US 8653094, 2014.

[7] (a) Ohkuma, T.; Koizumi, M.; Doucet, H.; Pham, T.; Kozawa, M.; Murata, K.; Katayama, E.; Yokozawa, T.; Ikariya, T.; Noyori, R. J. Am. Chem. Soc. 1998, 120, 13529.

(b) Kuroki, Y.; Asada, D.; Iseki, K. Tetrahedron Lett. 2000, 41, 9853.

(c) Kuroki, Y.; Sakamaki, Y.; Iseki, K. Org. Lett. 2001, 3, 457.

(d) Sterk, D.; Stephan, M.; Mohar, B. S.; Szollosi, G.; Bartok, M. Appl. Catal. A: Gen. 2009, 362, 178.

(f) Pereniguez, R.; Santarossa, G.; Mallat, T.; Baiker, A. J. Mol. Catal. A: Chem. 2012, 365, 39.

[8] (a) Gaspar, J.; Guerrero, A. Tetrahedron: Asymmetry 1995, 6, 231. (b) Nakamura, K.; Matsuda, T.; Itoh, T.; Ohno, A. Tetrahedron Lett. 1996, 37, 5727.

(c) Nakamura, K.; Matsuda, T.; Shimizu, M.; Fujisawa, T. Tetrahedron 1998, 54, 8393.

(d) Inoue, K.; Makino, Y.; Itoh, N. Tetrahedron: Asymmetry 2005, 
16, 2539 .

(e) Borzecka, W.; Lavandera, I.; Gotor, V. J. Org. Chem. 2013, 78, 7312.

[9] (a) Yamaguch, S.; Mosher, H. S. J. Org. Chem. 1973, 38, 1870. (b) Pirkle, W. H.; Sikkenga, D. L.; Pavlin, M. S. J. Org. Chem. $1977,42,384$

(c) Hawkins, J. M.; Sharpless, K. B. J. Org. Chem. 1984, 49, 3861. (d) Chong, J. M.; Mar, E. K. J. Org. Chem. 1991, 56, 893.

[10] (a) Stepanenko, V.; De, J. M.; Correa, W.; Guzman, I.; Vazquez, C.; Cruz, W.; Ortiz-Marciales, M.; Barnes, C. L. Tetrahedron Lett. 2007, 48, 5799 .

(b) Korenaga, T.; Nomura, K.; Onoue, K.; Sakai, T. Chem. Commun. 2010, 46, 8624 .

(c) Kawanami, Y.; Hoshino, K.; Tsunoi, W. Tetrahedron: Asymmetry 2011, 22, 1464.

(d) Turgut, Y.; Azizoglu, M.; Erdogan, A.; Arslan, N.; Hosgoren, H. Tetrahedron: Asymmetry 2013, 24, 853.

(e) Harauchi, Y.; Takakura, C.; Furumoto, T.; Yanagita, R. C.; Kawanami, Y. Tetrahedron: Asymmetry 2015, 26, 333.

[11] (a) Nasipuri, D.; Bhattacharya, P. K. J. Chem. Soc., Perkin Trans. 1 1977, 576.

(b) Morrison, J. D.; Tomaszewski, J. E.; Mosher, H. S.; Dale, J.; Miller, D.; Elsenbaumer, R. L. J. Am. Chem. Soc. 1977, 99, 3167.

(c) Yong, K. H.; Chong, J. M. Org. Lett. 2002, 4, 4139.

[12] Sasaki, S.; Yamauchi, T.; Kubo, H.; Kanai, M.; Ishii, A.; Higashiyama, K. Tetrahedron Lett. 2005, 46, 1497.

[13] (a) Yearick, K.; Wolf, C. Org. Lett. 2008, 10, 3915.

(b) Genov, M.; Martinez-Ilarduya, J. M.; Calvillo-Barahona, M.; Espinet, P. Organometallic 2010, 29, 6402.

(c) Calvillo-Barahona, M.; Cordovilla, C.; Genov, M. N.; Martinez-Ilarduya, J. M.; Espinet, P. Dalton Trans. 2013, 42, 14576.

(d) Calvillo-Barahona, M.; Casares, J. A.; Cordovilla, C.; Genov,
M. N.; Martinez-Ilarduya, J. M.; Espinet, P. Chem.-Eur. J. 2014, 20 , 14800 .

[14] Huang, H.; Zong, H.; Bian, G. L.; Song, L. Tetrahedron: Asymmetry 2015, 26, 835 .

[15] (a) Forni, A.; Moretti, I.; Prati, F.; Torre, G. Tetrahedron 1994, 50, 11995.

(b) Asao, N.; Asano, T.; Yamamoto, Y. Angew. Chem., Int. Ed. 2001, 40, 3206.

(c) Hess, R.; Diezi, S.; Mallat, T.; Baiker, A. Tetrahedron: Asymmetry 2004, 15, 251.

(d) Diezi, S.; Hess, M.; Orglmeister, E.; Mallat, T.; Baiker, A. Catal. Lett. 2005, 102, 121

(e) Grau, B. T.; Devine, P. N.; DiMichele, L. N.; Kosjek, B. Org. Lett. 2007, 9, 4951.

[16] (a) Huang, H. Y.; Zong, H.; Bian, G. L.; Song, L. J. Org. Chem. 2012, 77, 10427.

(b) Zong, H.; Huang, H. Y.; Bian, G. L.; Song, L. Tetrahedron Lett. 2013, 54, 2722.

(c) Shen, B.; Huang, H. Y.; Bian, G. L.; Zong, H.; Song, L. Chirality 2013, 25, 561 .

(d) Huang, H. Y.; Zong, H.; Shen, B.; Yue, H. F.; Bian, G. L.; Song, L. Tetrahedron 2014,70, 1289.

(e) Yue, H. F.; Huang, H. Y.; Zong, H.; Bian, G. L.; Zong, H.; Li, F. L.; Song, L. Tetrahedron: Asymmetry 2014, 25, 170.

[17] Hatano, M.; Miyamoto, T.; Ishihara, K. Org. Lett. 2007, 9, 4535

[18] Huang, H. Y.; Zong, H.; Bian, G. L.; Song, L. J. Org. Chem. 2015, $80,12614$.

[19] González-Martínez, D.; Gotor, V.; Gotor-Fernández, V. ChemCatChem 2019, 11, 5800.

[20] Brüning, F.; Nagae, H.; Käch, D.; Mashima, K. I.; Togni, A. Chem.-Eur. J. 2019, 25, 10818. 